\title{
Interplay between confinement, twist elasticity and intrinsic chirality in micellar lyotropic nematic liquid crystals
}

Clarissa F. Dietrich ${ }^{1}$, Per Rudquist ${ }^{2}$, Peter J. Collings ${ }^{3,4}$, Frank Giesselmann ${ }^{1, *}$

${ }^{1}$ Institute of Physical Chemistry, University of Stuttgart, 70569 Stuttgart, Germany.

${ }^{2}$ Microtechnology and Nanoscience, Chalmers University of Technology, 41296 Göteborg, Sweden.

${ }^{3}$ Department of Physics \& Astronomy, Swarthmore College, Swarthmore, PA 19081, USA.

${ }^{4}$ Department of Physics and Astronomy, University of Pennsylvania, Philadelphia, PA 19104, USA.

Supporting Information 
Experimental details: sample holders, water as index matching fluid
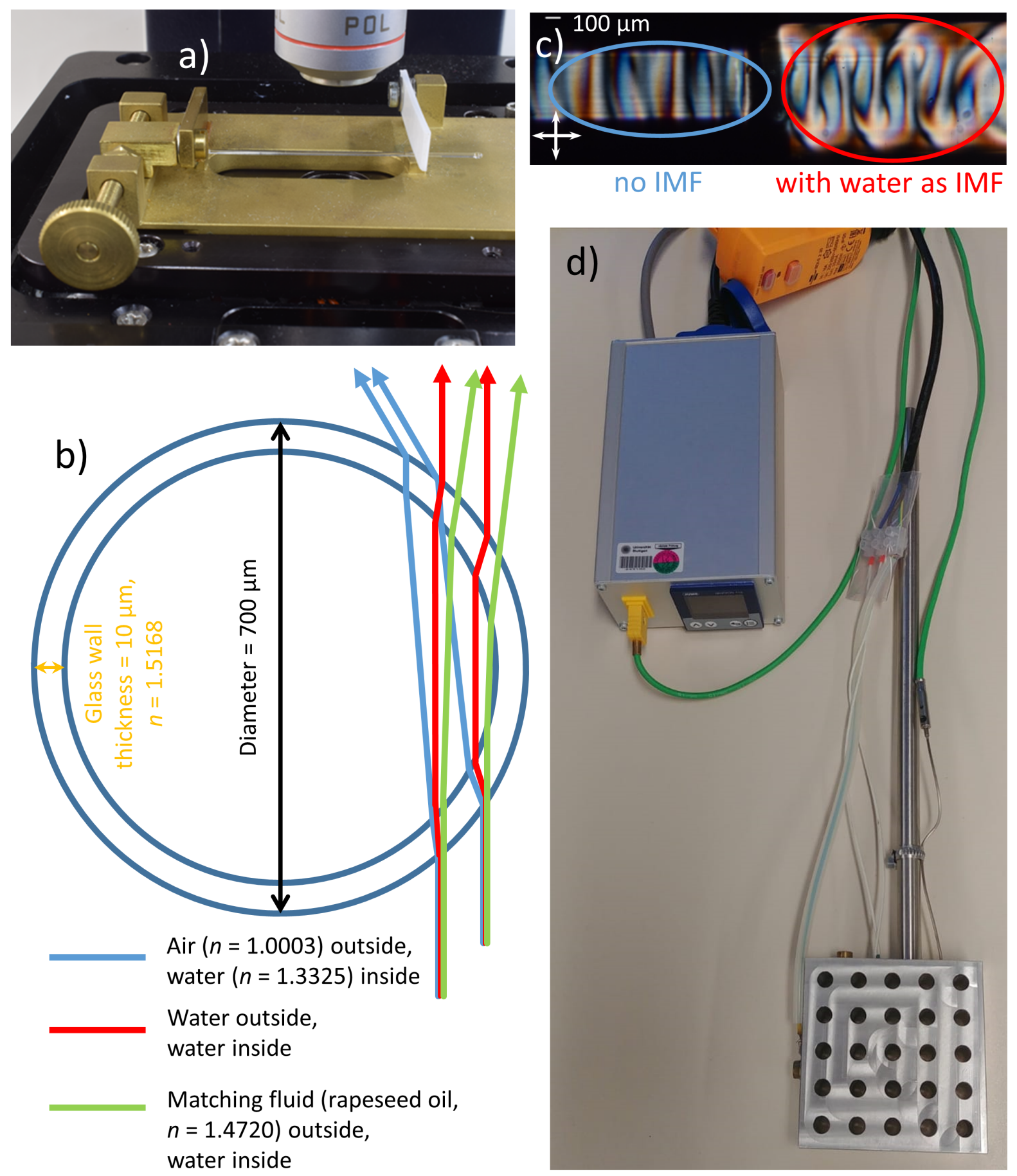

Figure S1. a) Sample holder used for rotating the capillaries under the microscope. b) Schematics of the action of an index matching fluid (IMF) when observing a glass cylinder filled with the lyotropic LC under the optical microscope. Without the index matching fluid (blue rays) or when the surrounding fluid has the same index as the glass (e.g., rapeseed oil, green rays), a significant part of the texture is not visible due to refraction effects. The largest field of view of the interior is obtained when the surrounding medium has the same refractive 
index as the LLC inside the capillary, which is approximately the case for water (red rays). c) Photographs of a $700 \mu \mathrm{m}$ diameter capillary without water (left) and with water (right) as the index matching fluid (IMF). d) Temperature-controlled sample holder for the magnetic field experiments.

\section{The twist disclination, transverse twist, and axial twist of the TP configuration}
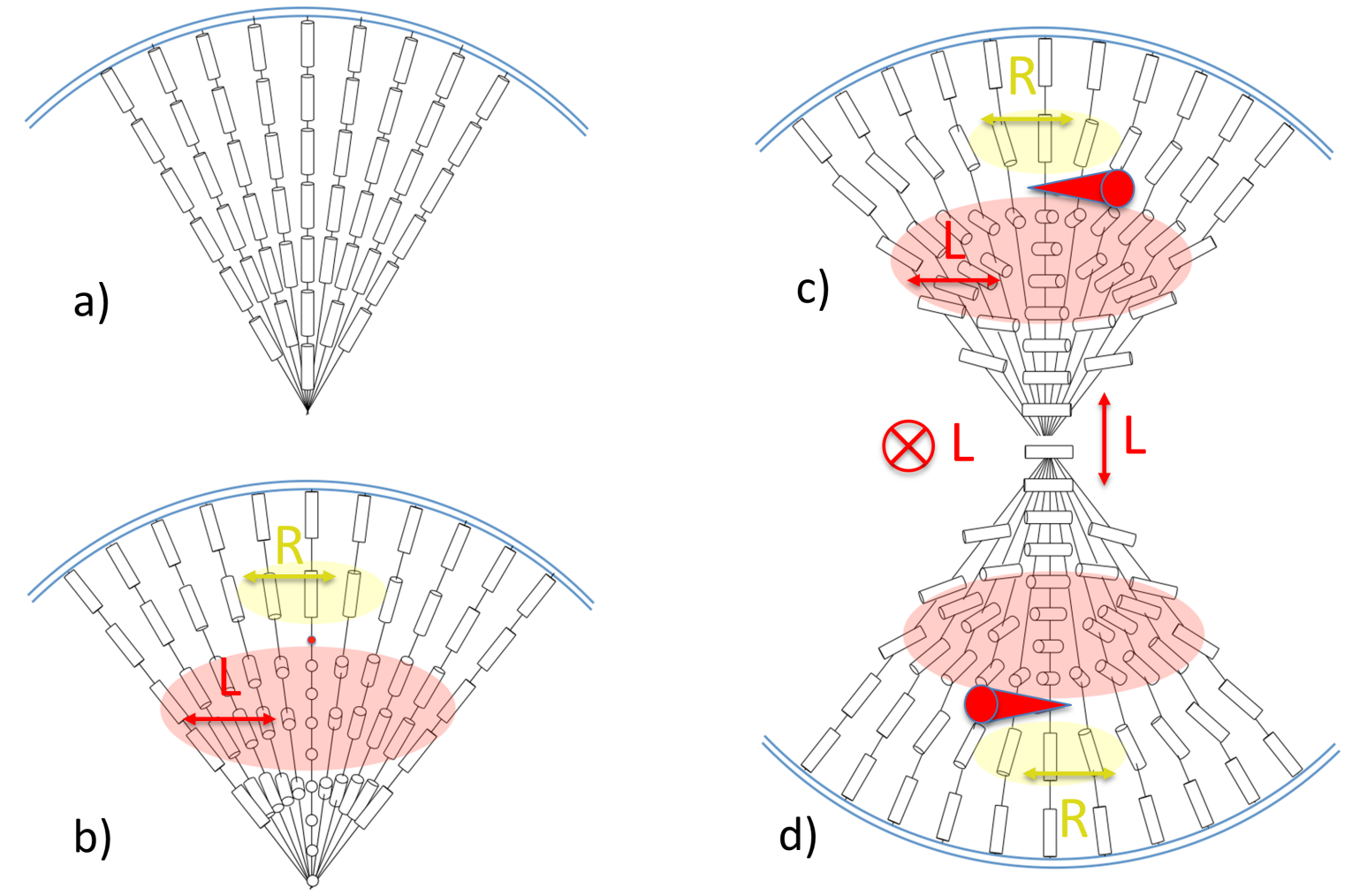

Figure S2. In order to visualize the relation between the twist disclination and the TP helix, we can do the following thought experiment. Let us imagine a cylinder with homeotropic boundary conditions filled with a nematic LC, where the director is represented by cylinders. a) First let the director be radial everywhere, which gives $a+1$ defect at the center. $b$ ) We then introduce $a$ $1 / 2$ unit twist disclination (red), normal to the plane of the paper, where the director makes a $180^{\circ}$ left-handed twist below the disclination, cf. S2b (pink area). Above the disclination a small region of weakly right-handed twist likely forms (yellow area).c) We then let the new director field relax into the TP structure. In addition to the left-handed twist from the twist disclination, both a left-handed (transverse) twist along the cylinder diameter and a left-handed twist along the cylinder axis form. The $1 / 2$ disclination line is no longer normal to the plane of the paper but slightly rotated away from the normal. d) If we imagine a similar disclination on the opposite side of the capillary, we can easily see that the two disclinations now form a double helix along the capillary. When adding a chiral dopant supporting left-handed twist, the right-handed twist 
region disfavored by the dopant (yellow), is likely pushed out from the volume, making the disclinations approach the capillary surface. This is in fact observed in our system as described in the main text.
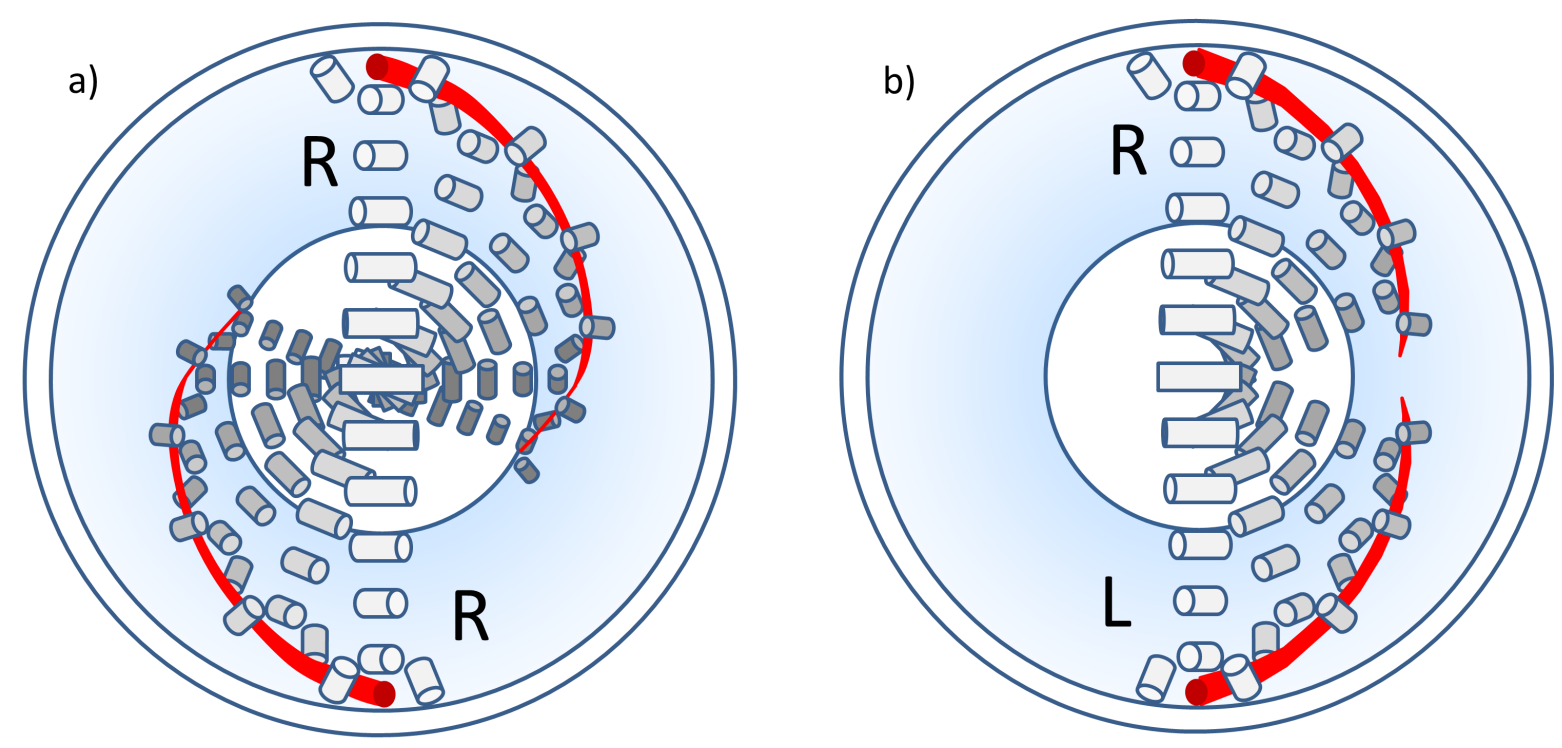

Figure S3. Illustration of the disclination lines (red) when the twist disclinations have (a) the same chirality (RR) and (b) opposite chirality (RL). In (a) the two lines spiral in the same direction and form a perfect double helix along the cylinder with maximum azimuthal distance between the lines. Hence, elastic energy is independent of the position along the cylinder. In (b) the lines spiral in opposite directions and approach each other when moving along the cylinder. Hence, the minimum azimuthal distance between the lines decreases which rapidly increases the elastic energy. By flipping the chirality of the lines, the azimuthal distance increases when continuing along the capillary and the elastic energy is lowered again. The process is repeated on the other side of the vertical diameter. In actuality, the flipping of chirality occurs for a small decrease in azimuthal distance, and the kinked disclinations stay close to the vertical diameter in (b). 